\title{
Supervised parameter estimation for road vehicles, mitigating powertrain induced uncertainty
}

DOI:

10.1109/TVT.2019.2949636

\section{Document Version}

Accepted author manuscript

Link to publication record in Manchester Research Explorer

\section{Citation for published version (APA):}

Wragge-Morley, R. T., Herrmann, G., \& Barber, P. (2020). Supervised parameter estimation for road vehicles, mitigating powertrain induced uncertainty. IEEE Transactions on Vehicular Technology, 69(7).

https://doi.org/10.1109/TVT.2019.2949636

\section{Published in:}

IEEE Transactions on Vehicular Technology

\section{Citing this paper}

Please note that where the full-text provided on Manchester Research Explorer is the Author Accepted Manuscript or Proof version this may differ from the final Published version. If citing, it is advised that you check and use the publisher's definitive version.

\section{General rights}

Copyright and moral rights for the publications made accessible in the Research Explorer are retained by the authors and/or other copyright owners and it is a condition of accessing publications that users recognise and abide by the legal requirements associated with these rights.

\section{Takedown policy}

If you believe that this document breaches copyright please refer to the University of Manchester's Takedown Procedures [http://man.ac.uk/04Y6Bo] or contact uml.scholarlycommunications@manchester.ac.uk providing relevant details, so we can investigate your claim.

\section{OPEN ACCESS}




\title{
Supervised parameter estimation for road vehicles, mitigating powertrain induced uncertainty.
}

\author{
Robert Wragge-Morley, Guido Herrmann, Sen. Member, IEEE, and Phil Barber
}

\begin{abstract}
This paper demonstrates a real world case study of a new method for robust simultaneous estimation of two parameters using multiple data sources. The method is used to simultaneously estimate vehicle mass and road gradient. No additional sensors are required beyond those that would normally be found on a vehicle controller network. The estimation algorithm combines components driven by observer state error and also directly by the parameter error using a sliding-mode inspired regressor structure. The algorithm incorporates a novel information fusion method that is integral to the regressor structure and a supervised data-rejection system to limit estimation activity in periods of recognised error-promoting activity. The estimation method has been demonstrated in real time on a modified production passenger car platform. It has been shown to be effective at robustly predicting road gradient and offering more reliable and stable prediction of vehicle mass than existing estimation methods employed in the same multi-parameter estimation context. The estimator allows prediction of vehicle mass whose limiting factor is the bandwidth and accuracy of the available driveline torque information, not the algorithm itself, allowing the identification of a $150 \mathrm{~kg}$ change in mass on a $2000 \mathrm{~kg}$ vehicle in this case study.
\end{abstract}

Index terms: Control Engineering, Land Vehicles, Parameter Estimation, Supervisory Control, System Identification

\section{INTRODUCTION}

In a world of increasingly autonomous and connected personal transport solutions, vehicle self-awareness is an ever-more-pressing challenge to the automotive engineer. In order to implement effective Advanced Driver Assist Systems (ADAS), more, and more detailed, knowledge of the vehicle system under control is required. The scope and goals of autonomy in vehicular systems are both broad and far-reaching, from improving the straightforward automated control of mechanical systems to completing complex learning and artificial intelligence tasks making up a fully autonomous driving system. At the most basic level of automating control tasks in the vehicle powertrain and deploying now commonplace ADASs such as cruise control, there is still room for improving both the perceived effectiveness of the system and the measurable performance. Systems in this category, as well as in the fully autonomous

R. Wragge-Morley is with the Powertrain and Vehicle Research Centre, Department of Mechanical Engineering, University of Bath, BA2 7AY Somerset, U.K. email: R.T.Wragge-Morley@bath.ac.uk

G.Herrmann is with the Department of Electrical and Electronic Engineering, University of Manchester

P.Barber has been with Jaguar Land Rover Chassis Research

Manuscript received August 22, 2018; revised December 15, 2018; revised April 03, 2019; revised July xx 2019 future of automotive control have the combined targets of improving vehicle efficiency, reducing environmental impact and increasing occupant and third-party safety. The majority of safety and energy management themed ADASs are systems designed to control either the lateral or longitudinal dynamic behaviour of the vehicle, or the coupled behaviour of both. In order to implement more effective real-time control, it is beneficial to have the most accurate model of the vehicle to be used for control.

One of the most fundamental parts of the vehicle model is the vehicle mass, since vehicle inertia is a substantial part of the dynamics of the vehicle system in all degrees of freedom. The engineering problem presented in this paper is concerned with the real-time estimation of vehicle mass; throughout the research work reported herein, a key goal was to avoid the fitment of additional sensors that would not normally be present in a current production passenger car. The estimation method focuses on using the vehicle powertrain as a data source for mass estimation.

As will become apparent from the estimation algorithm derivation in section II-A, it is always necessary to know certain other parameters of the longitudinal vehicle dynamics in order to compute vehicle mass from this system. All the losses involved in the dynamics are coupled in the longitudinal dynamics force balance by Newton's second law. One of the most significant unknown loss parameters in the mass estimation problem is the road gradient, knowledge of which is crucial to effective estimation of the vehicle parameters. Vehicle mass and road gradient are commonly estimated together since the ability to effectively determine one is highly dependent on knowledge of the other. Many of the studies in this area make use of the differing speed dynamics of these two parameters in order to employ multiple estimators or types of estimator. Kidambi [5] showed the performance potential of a number of different types of estimator, including recursive least squares (RLS), extended Kalman filtering (EKF) and a method including an accelerometer as an extra data source. This study made use of half-shaft torque transducers to accurately measure driving torques, thus greatly improving the accuracy of the estimation when compared to methods using powertrain torque estimates only.

For example, Raffone [17] uses a Kalman Filter for the relatively fast road gradient estimation and an RLS approach to determine the vehicle mass, which will be close to a 
constant when the vehicle is underway. Vahidi et al. [27] demonstrate how multiple forgetting factors can be used in an RLS with forgetting, in their case study a similar approach is taken by using different assumed covariances to represent the dynamics of the two parameters in a Kalman Filter, however this is outperformed by their RLS formulation. Similarly, Li, Zhang et al. [8] use an adaptive observer and adaptive law with fast and slow adaptation behaviours to the same end. Nonlinear observer structures are employed by McIntyre et al. [12] and Rajamani et al. [19] for vehicle parameter estimation.

Some methods are used repeatedly by different researchers in the estimation of parameters in vehicle longitudinal dynamics. Lingman and Schmidtbauer [9] presented a method based on an Extended Kalman Filter, with additional states for both vehicle mass and road gradient (in addition to those for the longitudinal vehicle dynamics), and Kalman Filtering is often used for the estimation of both individual and multiple parameters. For example, the work of Sahlholm et al. [21][22][4] regards the gradient as a system state, in order to allow the use of a Kalman Filter for road gradient estimation.

Throughout the parameter error driven method presented in this paper, road gradient is treated as a changing vehicle parameter, rather than as a state or an input as in some other research in this field. Any Kalman filtering based estimator would require the estimated properties to be formulated as states, this is true of vehicle mass as well as road gradient. Where comparisons are made between estimation methods, this is the approach taken.

In order to improve on the robustness of existing estimation algorithms, the authors of this paper propose a method recently developed by $\mathrm{Na}$, Mahyuddin and Herrmann $[14][15][10][11]$ at Bristol that combines several previously deployed estimation techniques developed to improve adaptive control and estimation. The core of the estimation algorithm is comprised of two parts. A gradient descent part is driven by the estimated-state-error of an observer for the vehicle dynamics. This is combined with finite and exponential time converging, sliding-mode inspired, regressor components driven by the actual parameter error. These structures are described in sections II-C and II-D respectively. In addition, a novel data fusion method using the regressor and a supervisory element managing input data are included in the algorithm. This latter technique forms one of the major contributions of the research involved in applying the parameter-error driven algorithm to a practical system. The rationale for these elements of the algorithm are given in the subsequent paragraphs and a full derivation is given in sections II-B to II-F. The core of the algorithm also includes a forgetting component in order to avoid colouration by historical data that has become insignificant.

There are certain shortcomings of being limited to the powertrain data, particularly related to the estimation of some values by the various subsystem control units and available accuracy of data. These difficulties will be described and addressed briefly in section III-D of this paper and the rationale by which they are overcome and challenges and opportunities therein form another major contribution of this work. In order to increase the robustness of the multi-parameter estimation algorithm to high uncertainty powertrain and braking system data, it is highly beneficial to include other data sources where they are available. In the estimation algorithm presented in this paper, longitudinal accelerometer from the anti-lock braking and airbag control module is included as an additional longitudinal data source.

There are significant precedents for using multiple information sources and data fusion techniques in the estimation of vehicle parameters. Caron et al. [1] and Sukkariah et al. [24] both combine data from inertial and GPS measurement. Peng et al. [16] use multiple inertial measurement systems. Rajamani et al. [19], and Kim et al. [6] both use roll dynamics to support a mass estimation algorithm and propose inertial measurement as a data source, whilst Reineh et al. [20] uses roll dynamics for the detection of roof mounted payloads.

There are some other more heuristic approaches in the literature for overcoming the shortfalls of the available powertrain data. For example, Ghosh et al.[3] build on the former work of the present author [26], among others, to limit estimator activity to the period of time when the vehicle is moving. The method presented by this author also includes a transmission observer and filtering arrangement to overcome the problems presented by low frequency dominated longitudinal dynamics. Fathy et al. [2] also isolated specific vehicle dynamics events to ensure good estimator performance for mass, with an emphasis on avoiding gear-shifts. The idea of using a supervisor in adaptive control is best described in I-D. Landau's book [7], which illustrates the need for a complete change of control strategy for certain plant behaviours, especially where a particular system turns on or off or experiences a step change.

In addition to including data from more than one source in the estimation process, the proposed algorithm increases its robustness to corruption caused by certain real driving events by including a supervisory system to manage the input data. This system is described in section III and the reasoning behind its design and implementation is explored. When combined with the forgetting behaviour in the main part of the algorithm, this method ensures that only relevant, current data are used for estimation.

The performance delivered can be demonstrated to practically and analytically be more robust and effective in differentiating between vehicles of differing weights given the same data sources than the methods employing the existing state of the art techniques such as recrusive least squares and Kalman filters. This clearly illustrates the potential of these new methods in light of ever improving measurement and estimation technology elsewhere in the vehicle system. 


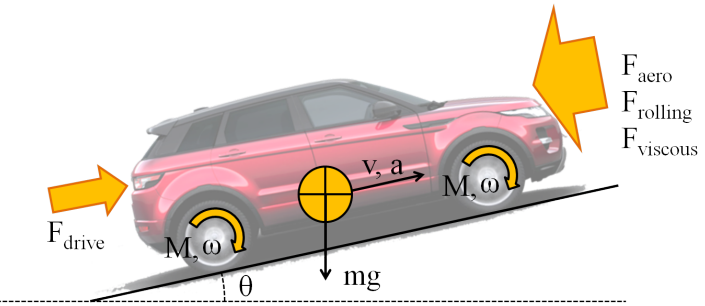

Fig. 1: Schematic of basic longitudinal vehicle dynamics

Furthermore, the methods could be employed for different systems of dynamics, to estimate different parameters. A discussion of the results is given in section IV, and a summary of the practical system employed is presented in section III-D.

\section{TECHNICAL BACKGROUND}

Much of the derivation of the estimation algorithm itself will not be presented here, only those parts that are key to the reader's understanding of the modifications made to it by the present author. The estimator has been modified in order to perform data-fusion from multiple sources and this is introduced.

\section{A. Vehicle Dynamics Parameterisation}

An illustration of the longitudinal dynamics of a road vehicle is given in Figure 1. The Newtonian force balance for vehicle longitudinal force is given in equation (1):

$$
\begin{gathered}
m \ddot{x}=F_{\text {drive }}-\overbrace{m g \sin \theta_{R}}^{\text {RoadGradient }}-\overbrace{C_{\mu} \cos \theta_{R}}^{\text {RollingResistance }} \\
-\underbrace{C_{V F} \dot{x}}_{\text {SpeedDependentLoss }}-\underbrace{\frac{1}{2} \rho C_{d} A_{f} \dot{x}^{2} \text { sgn } \dot{x}}_{\text {Speed }{ }^{2} \text { dependentloss }}
\end{gathered}
$$

where the variable $x$ is vehicle displacement, vehicle mass is represented by $m, F_{\text {drive }}$ is the propulsive force experienced by the vehicle system, this is assumed to include braking force and account for any losses and inertias internal to the powertrain. The gravitational constant is $g$ and the road gradient is $\theta_{R}$. The resistance forces on the vehicle are captured in several other terms. These are:

1) A rolling resistance term proportional to the cosine of road gradient. The rolling resistance coefficient is $C_{m} u$

2) A viscous friction term representing losses proportional to velocity, with the coefficient $C_{V F}$.

3) An aerodynamic term proportional to velocity squared, with the vehicle parameters for frontal area $A_{f}$ and drag coefficient $C_{d}$ and an environmental parameter for air density, which is assumed known (at sea level)

The values of vehicle parameters used in this work are proprietary values provided by the sponsor of the work and are determined from coast-down tests of a representative vehicle in a controlled experimental environment. A sign convention is used whereby forces, displacement states and derivatives thereof are considered positive in the direction of vehicle forward travel. The signum function $s g n$ is to prevent a sign error being introduced by the $\dot{x}^{2}$ term, in our case for the sake of efficiency, it is assumed that the vehicle only runs forwards to avoid issues related to forwards and backwards drag coefficients. This modelling approach is conventional and presented multiple times in well established literature [18] [13].

In order to derive the estimation algorithm, the vehicle dynamics must be arranged into a standard form state space equation. The structure of this equation is given in equation (2a) and its parameterised form in equation (3).

$$
\begin{gathered}
\dot{\boldsymbol{x}}=A \boldsymbol{x}+B_{1} \boldsymbol{u}_{1}+B_{2} f\left(x, u_{2}\right)+\zeta \\
y=C \mathbf{x} \\
\dot{\boldsymbol{x}}=A \boldsymbol{x}+B_{1} \boldsymbol{u}_{1}+B \boldsymbol{\varphi} \boldsymbol{\Theta}+\zeta
\end{gathered}
$$

where there are $n$ states, an $n \times n$ system matrix $A, n \times 1$ gain vectors $B_{1}$ and $B_{2}$ for the scalar input $\boldsymbol{u}_{1}$ and non-linear dynamics $f\left(\boldsymbol{x}, \boldsymbol{u}_{2}\right)$ and subject to $n \times 1$ noise vector $\zeta$. The measured states are contained in an $n \times 1$ vector $\boldsymbol{y}$ and the corresponding $n \times n$ measurement gain is matrix $C$, which is assumed for our current purposes to be an identity matrix and not subject to any additional noise. The non-linear dynamics $f\left(\boldsymbol{x}, \boldsymbol{u}_{2}\right)$ are parameterised using a vector of known (measured or implied) 'inputs' $\boldsymbol{u}_{2}$ and states $\boldsymbol{x}$ in $1 \times m$ vector $\boldsymbol{\varphi}$ and unknown, non-linearly varying parameters (to be estimated) in $m \times 1$ vector $\Theta$ shown in equation (3).

In the specific case of vehicle dynamics considered here and presented in equations (1), the states $\boldsymbol{x}=\left[\begin{array}{l}x_{1} \\ x_{2}\end{array}\right]$ are displacement and velocity, so that the dynamical system in equation (1) may be parameterised into the form given in (3). The known parameters and the unknown parameters (to be estimated) are given by equations (4a) and (4b) respectively. The known parameters are the gravitational constant and a term representing the resistance forces already described in equation (1) and grouped as per equation (5).The unknown parameters $s$ and $b$ represent the road gradient and vehicle mass parameters respectively. Equations (4d) and (4e) describe the two vectors isolating the second state (acceleration) dynamics and the system gains used to write the dynamics in the standard parameterised form given in (3).

$$
\begin{aligned}
\varphi & =\left[\begin{array}{ll}
-g & F_{n e t}
\end{array}\right] \\
\Theta & =\left[\begin{array}{c}
\frac{\sin \left(\theta_{R}+\tan ^{-1} C_{\mu}\right)}{\cos \left(\tan ^{-1} C_{\mu}\right)} \\
\frac{1}{m}
\end{array}\right]=\left[\begin{array}{l}
s \\
b
\end{array}\right] \\
A & =\left[\begin{array}{l}
A_{11}, A_{12} \\
A_{21}, A_{22}
\end{array}\right]=\left[\begin{array}{l}
0,1 \\
0,0
\end{array}\right] \\
B_{1} & =\left[\begin{array}{l}
0 \\
1
\end{array}\right] \\
B_{2} & =\left[\begin{array}{c}
0 \\
\bar{B}_{2}
\end{array}\right] \text { and } \bar{B}_{2}=1
\end{aligned}
$$




$$
F_{n e t}=F_{\text {drive }}-C_{V F} \dot{x}-\frac{1}{2} \rho C_{d} A_{f} \dot{x}^{2} \text { sgn } \dot{x}
$$

In order to perform certain analyses on the parameterised vehicle dynamics, it is convenient to further group the parameters. In this paper we shall define some further variable variable based on the second line of the vehicle dynamics state equation (6). By defining $\Psi$ to represent the known dynamics of the second state as a function of the state and known inputs, ie. $\Psi=f\left(x_{2}, u_{1}\right)$, and also defining a version of $\boldsymbol{\varphi}, \phi$ that includes the gain term $\bar{B}_{2}$, it is possible to rewrite equation (6) in a tidier form (7).

$$
\begin{aligned}
& \dot{x}_{2}=A_{21} x_{1}+A_{22} x_{2}+B_{12} u_{11}+\bar{B}_{2} \varphi \boldsymbol{\Theta}+\zeta_{2} \\
& \dot{x}_{2}=\boldsymbol{\Psi}+\boldsymbol{\phi} \boldsymbol{\Theta}+\zeta_{2}
\end{aligned}
$$

where:

$$
\begin{aligned}
\Psi & =A_{21} x_{1}+A_{22} x_{2}+B_{12} u_{11} \\
& =B_{12} u_{11} \\
\phi & =\bar{B}_{2} \varphi
\end{aligned}
$$

and $A_{21}, A_{22}$ and $B_{12}$ are components of the system matrices $A$ and $B_{1}$. Note that whilst the states $x_{1}$ and $x_{2}$ have values, $A_{21}$ and $A_{22}$ are null in the case of this system (see equation $(4 c))$, hence the reduced form of equation ( $8 b)$. The second element of the noise vector $\zeta$ is $\zeta_{2}$, and is generally assumed to be small, with most significant part of the vehicle dynamics being modeled.

\section{B. Estimation Algorithm Structure}

The detailed derivation of the composite parameter update law shall not be included here, since it has already been presented by the current authors and their colleagues in several places [14] [10] [11] [25] [26], however the structure of this update law is shown in equation (9) for the benefit of reader. The unique feature of this parameter estimation algorithm is that it permits well-defined finite-time or exponential convergence. It is robust to uncertainty and disturbances. This is achieved since the algorithm contains an element $R(t)$ directly dependent on the parameter estimation error:

$$
\dot{\hat{\boldsymbol{\Theta}}}=\Gamma[\underbrace{\varphi^{T} F(\boldsymbol{y}-C \hat{\boldsymbol{x}})}_{\text {Observer Error }}-\overbrace{R(t)}^{\text {Parameter Error }}]
$$

where the diagonals of $m \times m$ matrix $\Gamma$ contain the gains controlling the adaptation speed of the $m$ estimated parameters $\hat{\Theta}$. As elsewhere, $1 \times m$ vector $\varphi$ contains known parameters. The $m \times m$ matrix $F$ contains gains specific to the observer error driven part, whilst $n \times 1$ vectors $\boldsymbol{y}$ and $\hat{\boldsymbol{x}}$ are the measured and estimated states respectively. The measurement gain matrix is $C$. The $m \times 1$ vector $R$ represents the parametererror driven estimator structure which is repeated here in equations (11a) to (11c).

\section{Observer Error Driven Component}

The estimator is created to work with an observer. The structure of the non-linear adaptive observer is given in equation (10):

$$
\dot{\hat{\boldsymbol{x}}}=A \hat{\boldsymbol{x}}+B_{1} \boldsymbol{u}_{1}+B_{2} \boldsymbol{\varphi} \hat{\boldsymbol{\Theta}}+L(\boldsymbol{y}-C \hat{\boldsymbol{x}})
$$

observer error driven component uses a straightforward gradient descent algorithm with the error included in the observer via a Luenberger gain matrix $L$. However, since the gradient descent structure is prone to problems with robustness, we introduce the regressor structure detailed in the following section.

\section{Parameter Error Driven Component}

The parameter error driven regressor is represented in equation (9) by the term $R(t)$. Its general form is given in equation (11a) illustrating the finite and exponential time converging components whilst the formulation of the information matrix and vector $M$ and $N$ is given in equations (11b) and (11c) respectively:

$$
\begin{aligned}
& R(t)=\overbrace{M(t) \omega_{1} \Omega \frac{M(t) \hat{\boldsymbol{\Theta}}-N(t)}{\|M(t) \hat{\boldsymbol{\Theta}}-N(t)\|}}^{\text {FiniteTime }} \\
&+\underbrace{M(t) \omega_{2} \Omega(M(t) \hat{\boldsymbol{\Theta}}-N(t))}_{\text {ExponentialTime }} \\
& \dot{M}(t)=-k_{F F} M(t)+k_{F F} \boldsymbol{\phi}_{f}^{T}(t) \boldsymbol{\phi}_{f}(t), \\
& M(0)=0 \\
& \dot{N}(t)=-k_{F F} N(t)+k_{F F} \boldsymbol{\phi}_{f}^{T}(t) \underbrace{\left(\frac{x_{2}-x_{2 f}}{k}-\Psi_{f}\right)}_{\kappa}, \\
& N(0)=0
\end{aligned}
$$

where the scalar weights $\omega_{1}, \omega_{2}$ and the $m \times m$ positive definite diagonal matrix $\Omega$ govern the balance of the finite and exponential time converging components of the regressor and the relative adaptation speed for the two parameters in this part of the algorithm. The scalar $k_{F F}$ is a 'forgetting factor' whose weight effectively constrains the amount of historical data presently influencing the estimation output. Another system of filters is employed to remove the requirements to differentiate speed measurements numerically, hence we now consider the 'known' input parameters in $m \times 1$ vector $\phi_{f}$ rather than $\varphi$, although for practical purposes we may consider the information contained therein as being the same in this section; of greatest significance to the reader is that the $m \times m$ matrix $M$ contains information of the 'known' input parameters and that the $m \times 1$ vector $N$ contains information of the known parameters and measured signals in $\kappa$. A brief summary of the filtering rationale is given in the following section II-E: 


\section{E. Input Filtering}

From sections II-C and II-D, the reader may observe that the (time variant) known parameter input to the Parameter Error Algorithm is applied driving force and $F_{n e t}$ and that the corresponding state from straightforward Newtonian dynamics would be vehicle acceleration over ground. The available vehicle state, however, is velocity over ground and it is undesirable to take a numerical derivative of the digital signal. The solution to this, proposed by Mahyuddin (et al.) [10] [11] is to filter the grouped inputs to the algorithm defined in equations (8a) to (8c) according to the first order filter design in equations (12).

$$
\begin{aligned}
k \dot{x}_{2 f}+x_{2 f} & =x_{2}, & x_{2 f}(0) & =0 \\
k \dot{\Psi}_{f}+\Psi_{f} & =\Psi, & \Psi_{f}(0) & =0 \\
k \dot{\phi}_{f}+\phi_{f}, & =\phi & \phi_{f}(0) & =0 \\
k \dot{\zeta}_{2 f}+\zeta_{2 f} & =\zeta_{2} & \zeta_{2 f}(0) & =0
\end{aligned}
$$

This allows rearrangement of the expression for the filtered second-state (12a) to give an expression for filtered acceleration $\dot{x}_{2 f}$ in terms of measured and filtered speed over ground:

$$
\dot{x}_{2 f}=\frac{x_{2}-x_{2 f}}{k}
$$

For reference, the complete, filtered, system dynamics are presented in equation (14) below, this equation is the result of substituting equations (12b) to (13) into (7) and rearranging.

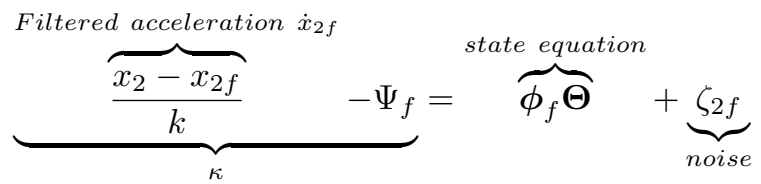

Consider the filtered system equation (14) for the second state, which is analogous to the relationship between $M, N$ and $\Theta$ shown in equation (16).

Pre-multiplying equation (14) by $\phi_{f}$ gives equation (15), below:

$$
\underbrace{\phi_{f}^{T} \kappa}_{N}=\underbrace{\phi_{f}^{T} \phi_{f}}_{M} \Theta+\boldsymbol{\phi}_{f}^{T} \zeta_{2 f}
$$

Observe the similarities between the terms labelled $N$ and $M$ in equation (15) and the design of the regressor vector and matrix shown in equation (11c) (11b). Thus for the case where $\zeta_{2}=0$, it follows from the regressor definition that:

$$
M(t) \hat{\boldsymbol{\Theta}}-N(t)=M(t) \tilde{\boldsymbol{\Theta}}
$$

where $\tilde{\boldsymbol{\Theta}}=\hat{\boldsymbol{\Theta}}-\boldsymbol{\Theta}$ is the error between the estimated and actual parameter values.

\section{F. Regressor-based Data Fusion Method}

Notice that it is possible to write other system dynamics as a parametrisation using the same 'unknown' parameters. A general form of such an expression is presented in equation (17):

$$
z=\varphi_{z} \Theta+\Psi_{z}+\zeta_{z}
$$

where $z$ is the state or state vector of these new dynamics, $\varphi_{z}$ is the 'known' part of the parametrisation of the new dynamics and $\Psi_{z}$ and $\zeta_{z}$ are the known linear dynamics and systematic noise respectively. A slight rearrangement of these terms allows us to group them in a way that is analogous to equation (14); of course, if incoming information is filtered for estimation based on the main system dynamics, then these additional dynamics $z$ must be filtered using filters in the same way. Grouping and filtering the expression for $\boldsymbol{z}$ gives the expression shown in equation (18) and allows us to construct a combined parametrisation in the form $\kappa=\phi \Theta$ with extended $\kappa$ and $\phi$ vectors shown in equation (19).

$$
\begin{aligned}
& \underbrace{z_{f}-\Psi_{z f}}_{\kappa_{z}}=\varphi_{z f} \Theta+\zeta_{z f} \\
& \underbrace{\left[\begin{array}{c}
\kappa \\
\kappa_{z}
\end{array}\right]}_{\kappa_{N}}=\underbrace{\left[\begin{array}{c}
\phi_{f} \\
\phi_{f z}
\end{array}\right] \Theta}_{\phi_{f N} \Theta}+\zeta_{N f}
\end{aligned}
$$

The regressor formulations remain the same as those in equations (11a) to (11c) but using $\kappa_{N}$ and $\phi_{f N}$ instead of the previous $\kappa$ and $\phi_{f}$ :

$$
\begin{gathered}
\dot{M}_{N}=-k_{F F} M_{N}(t)+k_{F F} \phi_{f N}^{T} \phi_{f N} \\
\dot{N}_{N}=-k_{F F} N_{N}(t)+k_{F F} \phi_{f N}^{T} \kappa_{N}
\end{gathered}
$$

Note that

$$
\begin{gathered}
M_{N} \hat{\Theta}-N_{N}(t)=M_{N} \tilde{\Theta}-\xi_{\phi N f} \\
\dot{\xi}_{\phi N f}=-k_{F F} \xi_{\phi N f}(t)+k_{F F} \phi_{f N}^{N} \xi_{\phi N f}(t)
\end{gathered}
$$

and $\tilde{\Theta}=\hat{\Theta}-\Theta$ and $\tilde{N}=M \hat{\Theta}-N(t)$. It should be noted that $\phi_{f N}$ is now a $p \times n$ matrix where $p$ is the number of sets of dynamics (or information sources) being considered.

\section{G. Comments on the stability of the Regressor Structure}

The theoretical basis, for Lyapunov stability of the estimator structure has been provided in previous works by $\mathrm{Na}$ et al. [15][14] and Mahyuddin et al. [11]; The theoretical proof for the regressor based data fusion method, building on [11][15][14], is provided as an exemplary proof in the Appendix A. This will also include the supervisor structure, as discussed in Subsection III-A.

However, practically it is vital to understand that the performance of the Parameter Error Algorithm is dependent on the numerical conditioning of the regressor structure. An assessment of this can be made by examining the regressor matrix $M$ (or $M_{N}$ ). The matrix must be invertible for the estimator to perform satisfactorily. The higher the condition number of the matrix $M$, the closer the matrix is to singularity and the more significant the detriment to the stability of the algorithm. In order to guarantee non-singularity of the matrix $M$, persistent excitation via the input vector $\varphi$ is required. This property was discussed at some length by Mahyuddin [10]. The condition number of a matrix is the ratio of the largest and smallest eigenvalues of that matrix, presented for $M$ in the following pseudocode. 


$$
\text { Condition }=\frac{\max \{\operatorname{eig}(M)\}}{\min \{\operatorname{eig}(M)\}}
$$

A low condition number is implicit of greater ease of invertability of the matrix $M$ and consequently greater stability of the parameter estimation algorithm.

From these conditions for estimator stability, the reader may note that periods of low excitation and low magnitude of some of the input parameters into the algorithm will have a severe negative effect on its performance. Later in the paper, when discussing supervisory control inspired estimation in section III-C, several sources of these numerical effects will be referred to.

\section{Contribution: Methods And Algorithms}

In order to deal with uncertainty in input data, a system regulating the Parameter Error Algorithm performance based on an assessment of the input data is required.

As has already been noted in this paper, the mass parameter being estimated should be effectively constant whilst the vehicle is in motion, since the rate of change in mass due to fuel consumption is very small and it is unlikely that the vehicle payload will change as the vehicle is moving, at least in the case of a passenger car. The implication of this is that one of the parameters being estimated is time varying whilst the other is not, although it is subject to change whilst the vehicle and estimator are inactive.

It is very important that the filtering parts of an estimation scheme are not allowed to prevent adaptation of a nearly constant parameter such as vehicle mass. It is a parameter which can and does change, albeit normally as a step change while the vehicle is static. Thus it is imperative that the estimator be allowed to adapt and converge rapidly on a true value of mass, at least early in the duty cycle under examination.

\section{A. Supervised Output Filtering}

A solution which has been used effectively to reduce noise on the mass estimate by the present author and others is the Kalman filter set up to estimate a constant parameter [26][17]. In the present context, considering the Parameter Error Algorithm, a supervised (i.e. part time) Kalman filter has been set up to manage the output of the mass estimate term. For the mass estimate, this filter is set up to adapt only when certain criteria regarding the current driving scenario are met. This avoids the output of the Parameter Error Algorithm becoming coloured by the recent ill-conditioned input data with the implications explained earlier in this section. In order to 'pause' the estimate, the current value is held constant until conditions improve, by setting all adaptation gains to zero temporarily when the estimator is required to pause. The structure of the estimator given in section II-B is represented graphically in Figure 2, with the output filter described in this section controlling the output of both states. As mentioned earlier, an exemplary discussion for stability of this supervised structure together with the

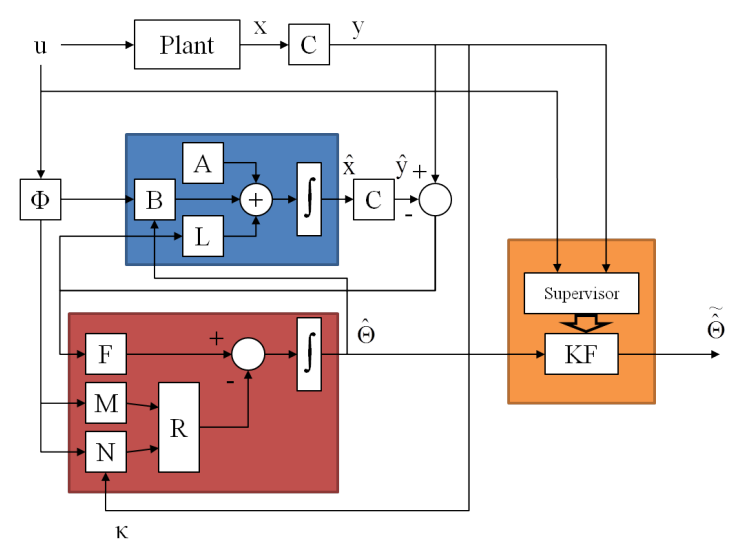

Fig. 2: Layout of Supervised Output Filtering in which the supervisor controls the speed and activity of the output filter

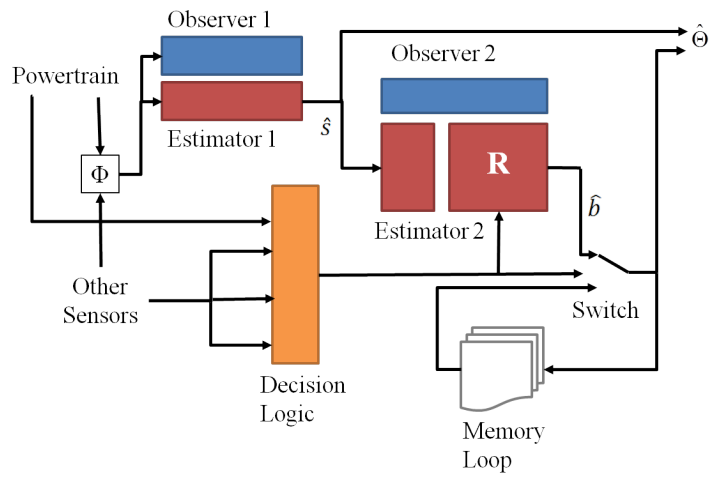

Fig. 3: Layout of in-series estimators with a supervisor applied to only a single parameter

parameter estimation system of Section II is provided in Appendix A.

\section{B. Supervised in-series estimators}

One approach for practically implementing the supervisory logic is to use two single-parameter estimators in series, with the gradient estimator operating continuously, based on an appropriately tuned mass parameter and a separate mass estimator taking the estimated gradient parameter as an input. The regressor part of the mass estimator being switched in and out of operation when conditions are favourable for its operation, and the last trusted value being used otherwise. In this way the mass estimate forgetting factors only process data from the selected time periods, whilst the forgetting factors in the gradient estimator correct for the error introduced in its mass parameter due to the richness of information from the data fusion technique. This scheme is illustrated in Figure 3.

\section{Supervisor Criteria}

Figure 4 illustrates how data from the measured variables and assumed known parameters are fed to the decision logic, which either activates or pauses the output filtering. The criteria may be mixed by way of switches for the purposes of 


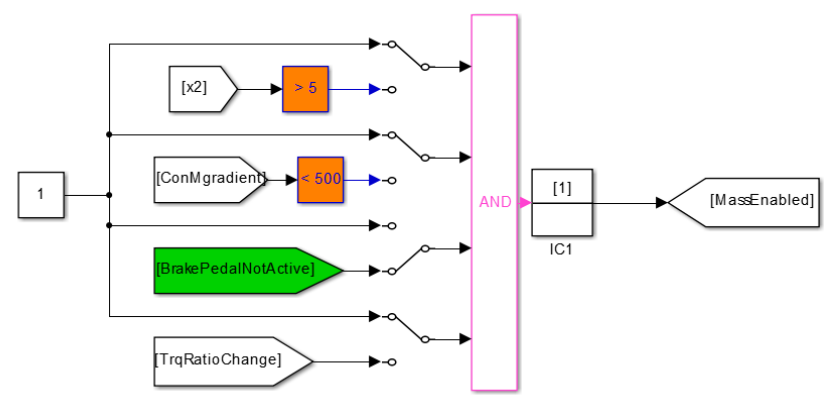

Fig. 4: Illustration of switch for mixing different enabling criteria in the estimator.

experimentation. The properties that are of particular interest for the practical estimation example examined in this paper and have been used in decision making are as follows:

Low Vehicle Speed-Over-Ground The significance of small numerical values of the input parameters is explained in section II-G, however there are several other reasons why it is not desirable to consider the outputs of powertrain dynamics driven estimator at very low speeds. Tyres are in their transient phase between their static and dynamic conditions and thus rolling resistance losses are not well defined by computationally practicable models. In addition, levels of slip in the torque converter are high out of necessity because the output shaft speed is close to zero but the engine is still turning at high speed. This last point is significant enough to warrant expansion further down this list.

Magnitude of driving force close to zero Since driving force is one of the two 'known' signals included in known parameter vector $\varphi$, which in turn forms the basis of regressor matrix $M$ and vector $N$, if the driving force is equal to zero, the stability of the regressor matrix is adversely affected, yielding poor results.

Vehicle friction brakes in use It has already been acknowledged elsewhere in this paper that friction brakes are difficult to model effectively due to the variety of external variables affecting their performance. When considering model validation, it was shown that whilst power-train dynamics models and collected data were able to agree to within $4 \%$ on the propulsive force required to drive a vehicle, even with relatively crude models in use, it was extremely unlikely that the reported braking effort would correlate with more than $15 \%$ accuracy. This is due mainly to the difficulty in reporting the conditions under which braking is carried out to the vehicles' onboard estimation systems.

Torque converter slip activity detected The torque converter or fluid coupling is part of the transmission system in the vehicles used for experimentation supporting this research. When the input shaft speed is greater than the output shaft speed, the torque is multiplied according to a speed-ratio dependent law. This behaviour, however, is affected by a number of variables, including temperature, load torque and properties of the hydraulic fluid being used; it is therefore difficult to accurately predict the transmission behaviour when the torque converter is slipping. In the case of a manual transmission or $\mathrm{DCT}^{1}$, where clutches are employed, non-linear uncertainties will still arise when the input and output shaft speeds are not proportional to one another - ie. when slip occurs. A comparison of the input and output shaft speeds, combined with information on current gear ratios, or an examination of the overall input to output shaft speed ratio for the entire transmission can provide information regarding the current slip behaviour of a torque converter or clutch, and thus be used to influence the decision process.

Condition number of regressor matrix $M$ This is a measure of the invertability of the regressor part of the novel estimation algorithm which gives an indication of the stability of the estimation algorithm given its current input prameters. A discussion of this is given in section II-G and a formulation for the condition number given in equation (23). Consistent with this assertion, it has been noted that the performance (of mass estimation results in particular) is better when the condition number of $M$ is low.

Of course, the above list is not exhaustive, and many of the additional dynamics of a vehicle may influence the performance of an estimator.

\section{Demonstration hardware}

A turn-key demonstration system was developed for the Parameter Error Algorithm and its derivatives. The system was installed in a Range Rover Evoque, kindly provided by the industrial sponsor of this research, JLR. The demonstrator was designed to allow rapid prototyping of new variations of the estimation algorithm and also to allow longer term data collection should this be necessary to the project.

The layout of hardware and communications buses used to implement the estimator is shown in figure 5, whilst the outputs were displayed in the vehicle cockpit by the small screen shown in figure 6. Data were read from the vehicle's $\mathrm{CAN}^{2}$ bus using the built in capability of the dSPACE MicroAutobox realtime computer on which the estimation trial was carried out. The vehicle was also fitted with an additional, high accuracy IMU ${ }^{3}$ and a GPS ${ }^{4}$ receiver which was used to provide a synchronising time pulse to the other hardware and as an independent speed over ground measurement.

In developing an effective, real world vehicle mass and road gradient demonstrator, a number of technical problems were overcome. These will be discussed later in the paper as important learning outcomes from this research.

\footnotetext{
${ }^{1}$ Dual Clutch Transmission

${ }^{2}$ Controller Area Network

${ }^{3}$ Inertial Measurement Unit

${ }^{4}$ Global Positioning System
} 


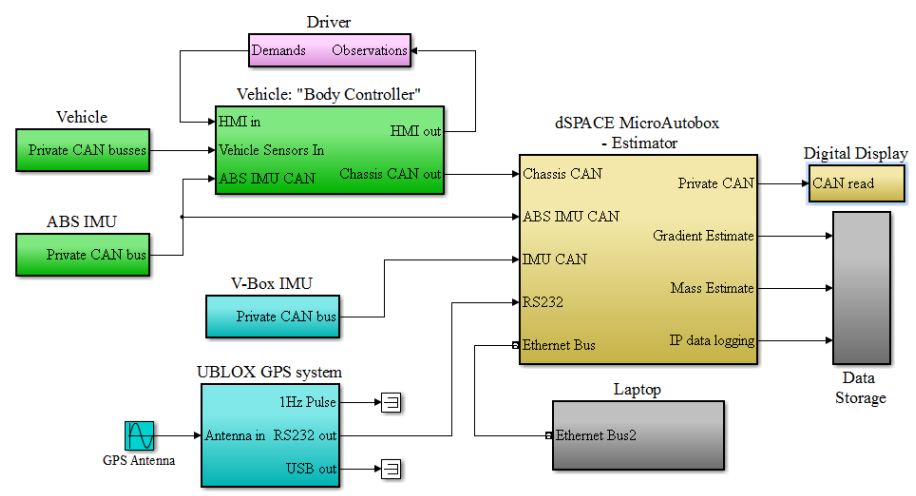

Fig. 5: Hardware layout for real time demonstration showing bus connections to the dSPACE and digital display (yellow), vehicle control hardware (green), extra sensing equipment (cyan) and data acquisition tools (grey).

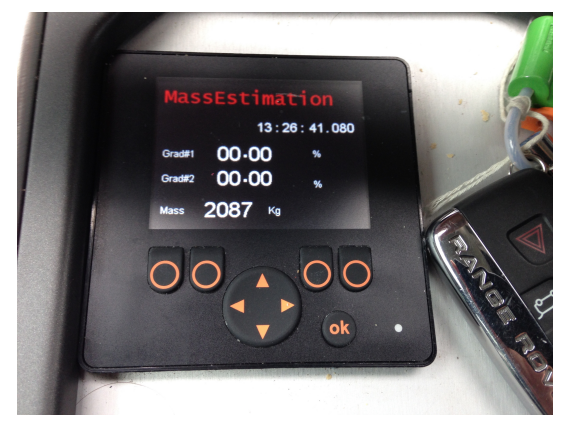

Fig. 6: Small digital display used for in-car experiments

\section{RESUlts}

\section{A. Simulated Results}

In a simulated experiment, a scaling was deliberately introduced on braking system data, with the mass estimation result shown in Figure 7. This effect was almost negated by the introduction of an appropriate supervisor design rejecting data from this source during problem periods, illustrated in Figure 7.

\section{B. Experimental Results}

Numerous practical studies were made of the performance of different versions of the Parameter Error Algorithm and supervisory and data fusion methods derived from it. Initially these consisted of off-line, desktop, processing based on sample data collected from real vehicles. Following the proof of concept of the algorithm, real-time testing was carried out on the vehicles.

1) Road Gradient Results: Testing was undertaken at Lommel Proving Ground in Belgium. A vehicle with a real-time computer and data-logging capability was used to test the Parameter Error Algorithm on the artificial hills. An example gradient estimation result superimposed on an aerial photograph of the test tracks is used to illustrate the effectiveness of the gradient estimation part of the algorithm in Figure 8. Most of the difficulties surrounding the

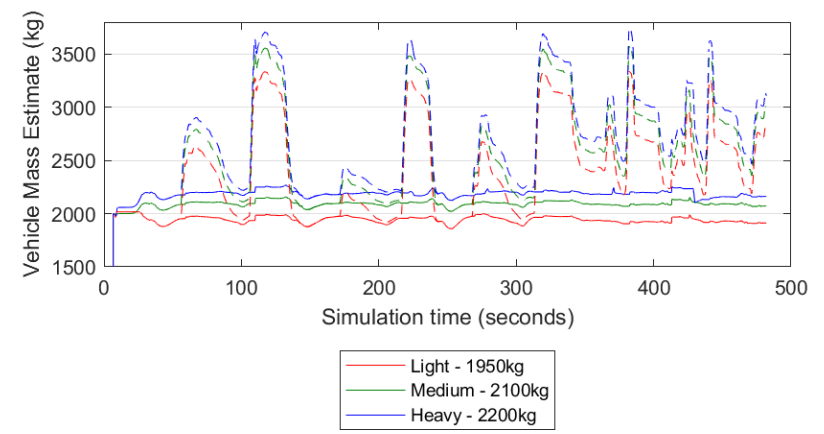

Fig. 7: Comparison between novel estimator result for a simulated plant where the reported driving force is deliberately multiplied 1.75 times during braking, with the regressor operating continuously and with the regressor supervised to reject data during braking events. The solid lines are for the supervised estimator and the dashed lines represent the unsupervised estimator.

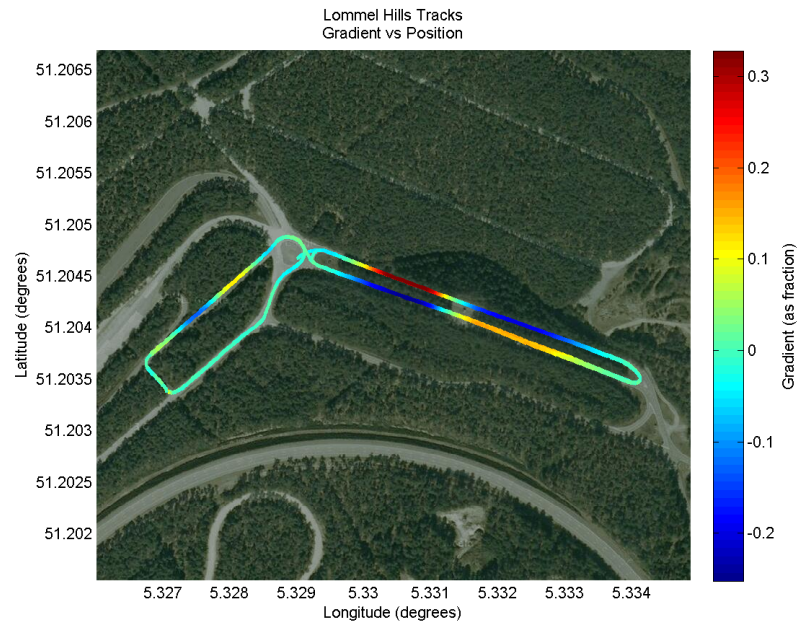

Fig. 8: Map of the artificial hills tracks at Ford's Lommel proving ground, with estimated road gradients superimposed from data collected on 14/08/2014

simultaneous estimation of vehicle mass and road gradient are dependent on the different speed dynamics of these two parameters. The gradient profile for the illustrated route is given in Figure 9; the large hills are driven in a clockwise loop 3 times with the sequence: $+30 \%,-20 \%,+15 \%,-25 \%$, followed by two counter-clockwise loops of the smaller hills.

2) Mass Estimate Results: The vehicle mass estimate performance achieved with the Parameter Error Algorithm exceeded that of appropriately designed estimators using existing state-of-the-art methods such as the Recursive Least Squares (RLS) Algorithm (see [23] and Appendix B) and the Kalman filter approach (see Appendix C). In a test consisting of driving the same duty cycle repeatedly with two different levels of payload, the Parameter Error Algorithm correctly distinguished between lightly and heavily loaded vehicles consistently based on the powertrain data input, which the 


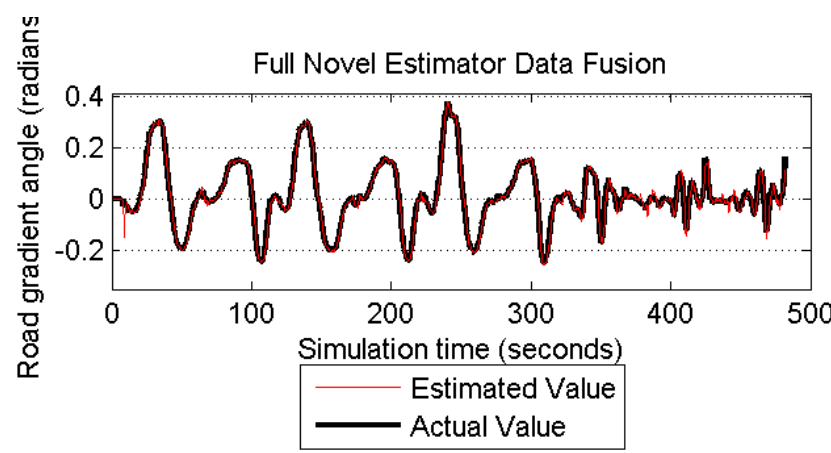

Fig. 9: Comparison of the performance of the full novel estimator with data fusion (equation (19)) to the actual road gradient input into simulated plant.

RLS and Kalman Filter based methods did not. These results are reported in table I, whilst the RLS and KF formulations employed are given in an appendix. This is in contrast to the simulated case where all methods performed similarly, illustrated in figure 10.

One of the limitations of any estimation algorithm is the accuracy of the input data. In the case of vehicle mass estimation, the changes in the parameter to be estimated are a small proportion of its total value. In contrast, when using powertrain data as an input, the uncertainties on torque delivered are very large in certain scenarios. Particular mechanical systems within the driveline and braking systems introduce phenomena that are difficult for the relevant existing control systems to account for when reporting the applied torque or force. Most of the uncertainty comes from the behaviour of either fluids or friction, in devices such as clutches, torque converters, tyres and disc brakes, all of which are dependent on at least one of these two broad phenomena in their operation. Full thermal and fluid models of any of these components are computationally very demanding and their performance is highly dependent on environmental factors such as ambient temperature. A further analysis of the specific problems encountered will be given, as appropriate, in section V.

The influence of the duty cycle on the vehicle's mechanical systems is very obvious in Figure 11; this illustration, along with those from the existing methods are plotted with distance travelled from the start of the duty cycle on the horizontal axis. Junctions and estimator behaviour that correlates to specific stretches of the route is visible, for example between 4600 and $6400 \mathrm{~m}$.

\section{DiscusSiOn}

In this paper, the problem of on-line estimation of vehicle parameters from powertrain data has been discussed. Simulated and experimental results have been presented focusing on the ability of different estimation techniques, including a novel Parameter Error based Algorithm , to determine the difference in mass between different payload conditions. Also introduced is the two-stage supervised
TABLE I: Comparison of the vehicle mass estimation results for six test runs on public highways near to the University of Warwick campus, where half of the runs are with $100 \mathrm{~kg}$ of ballast added to the vehicle. All masses are reported in $\mathrm{kg}$ and rounded to 3 significant figures for display. The data to which they refer are displayed in Figure 11. The mass values quoted in this table are averages over the settled period of estimation in each case, ignoring the initial adaptation.

\begin{tabular}{|c|c|c|c|}
\hline Test Run & PEA & RLS & KF \\
\hline \hline 1 & 2340 & 2490 & 1930 \\
4 & 2400 & 1950 & 1920 \\
5 & 2350 & 1950 & 2070 \\
\hline $\begin{array}{c}\text { Average of } \\
\text { heavy Runs }\end{array}$ & 2360 & 2130 & 1980 \\
\hline \hline $\begin{array}{c}2 \\
6\end{array}$ & 2290 & 2180 & 2010 \\
\hline $\begin{array}{c}\text { Average of } \\
\text { light Runs }\end{array}$ & 2250 & 2330 & 2050 \\
\hline \hline $\begin{array}{c}\text { Average } \\
\text { difference }\end{array}$ & 100 & -200 & -70 \\
\hline
\end{tabular}

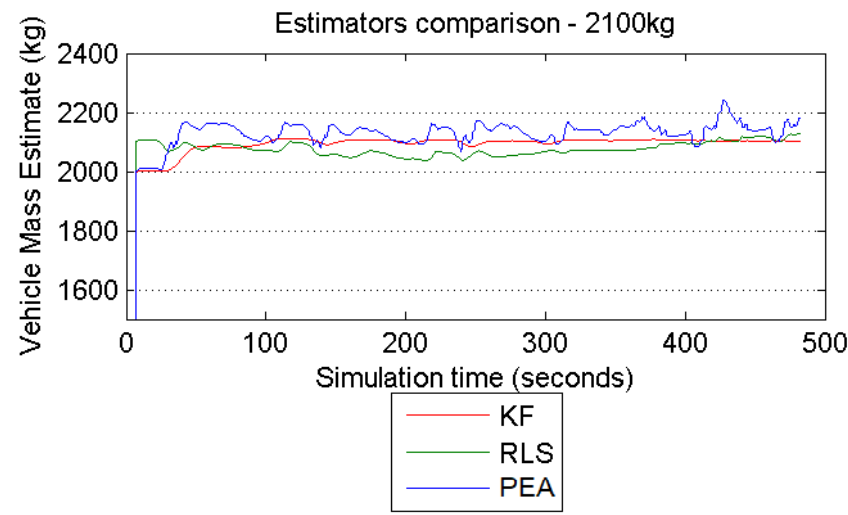

Fig. 10: Comparison of performance of different estimation methods in simulation where inputs to the estimator are well known

estimator structure.

A key difficulty evident from the real world experimental results is that estimation accuracy is dependent on the level of uncertainty on the powertrain's reported driving torque value. This, particularly during periods of engine and transmission transient behaviour can introduce substantial errors that become significant when the change in payload of a passenger entering or leaving the vehicle is only $3-4 \%$ of the total vehicle mass. A much better estimation result would be possible if, for instance, vehicles were fitted with half-shaft torque transducers, as this would remove nearly all of the internal disturbances and uncertainties, leaving only those from the environment. 


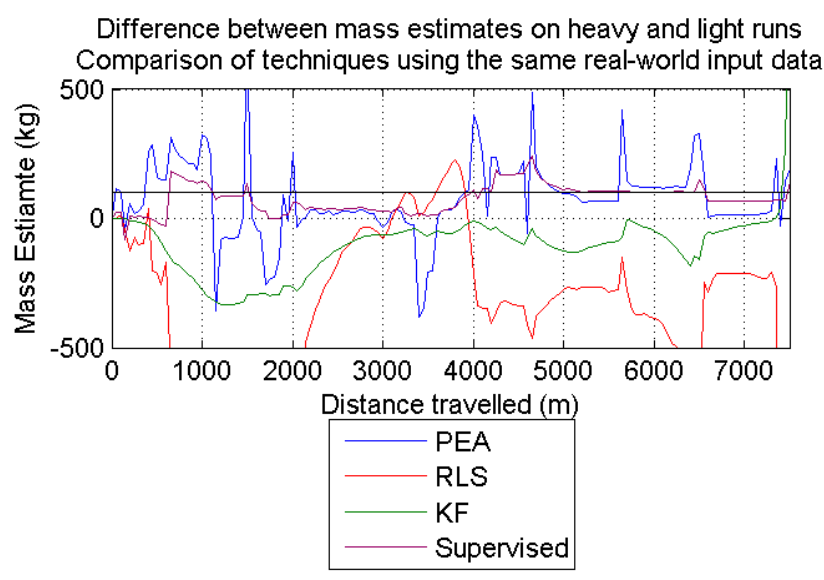

Fig. 11: Comparison of performance of different estimation methods using real-world powertrain data where the inherent uncertainties are great. The supervised output is rejecting data during friction-brake application.

\section{A. Numerical Condition of the Estimator}

One of the key contributions of the Parameter Error Algorithm is an increase in robustness and stability of the estimate. In section II-G, the 'condition number' of the regressor matrix is presented as an indication of the invertability of that matrix. This value gives an indication of how close to singularity the regressor is, the bigger the condition number, the closer and thus less stable. Since the closeness of this matrix to singularity is influenced directly by the level of excitation in the input data, it is undesirable to have limited or null input data into the algorithm. Both the supervisory structure introduced in section III and the data fusion method presented in section II- $\mathrm{F}^{5}$ seek to address this by reducing the tendency to process numerically problematic values and zero crossings and simultaneously increasing the level of excitation respectively. Figure 12 illustrates the reduction in condition number with the introduction of the data fusion method, representing an increase in stability of the Parameter Estimation Algorithm.

Examining equation 23, it is evident that another way of improving the stability of the regressor is scaling the inputs so that their values are closer in magnitude. The effect of disparity between the magnitude of the large and small values in the matrix $M$ scales with vehicle mass, and required driving force. This is illustrated in table II, below.

It is of course straightforward to introduce a scaling matrix to the parameterised form of the algorithm:

$$
\begin{aligned}
\boldsymbol{\varphi}_{g} & =\underbrace{\left[\begin{array}{cc}
g_{1} & 0 \\
0 & g_{2}
\end{array}\right]}_{G} \boldsymbol{\varphi} \\
\dot{\boldsymbol{x}} & =A \boldsymbol{x}+B_{1} \boldsymbol{u}_{1}+B G^{-1} \boldsymbol{\varphi}_{g} \boldsymbol{\Theta}+\boldsymbol{\zeta}
\end{aligned}
$$

so that $\varphi_{g}$ is the scaled version of $\phi$, and the matrix of scaling factors $g_{i}$ is $G$.

5 and the authors' previous work [25]

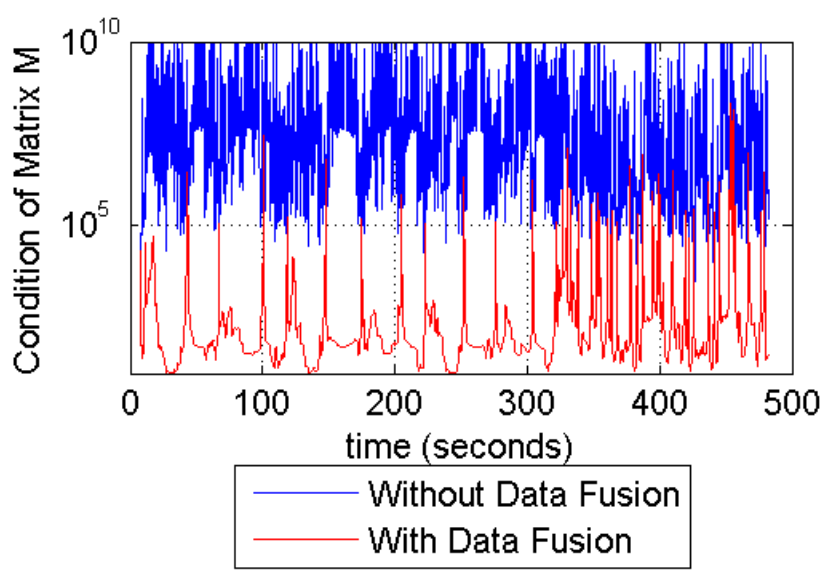

Fig. 12: Illustration of the change in the condition number of the matrix $M$ when using real data with and without data fusion active. The larger the number, the closer the matrix is to singularity and the worse the stability performance of the regressor will be.

TABLE II: The impact of changing the scale of the estimation problem on its stability illustrated using rough expected input magnitudes for an adequately excited system in each case. The rough orders of magnitude are a $10 \mathrm{~kg}$ lab vehicle being propelled by a $3 \mathrm{~N}$ force and a $2000 \mathrm{~kg}$ road vehicle being

\begin{tabular}{|c|c|c|c|}
\hline & M & $\mathrm{N}$ & $\hat{\Theta}$ \\
\hline & {$\left[\begin{array}{cc}g^{2} & -g F_{f} \\
-g F_{f} & F_{f}^{2}\end{array}\right]$} & {$\left[\begin{array}{l}-\dot{v}_{x} g \\
\dot{v}_{x} F_{f}\end{array}\right]$} & {$\left[\begin{array}{c}\sin \theta+C_{\mu} \cos \theta \\
\frac{1}{m}\end{array}\right]$} \\
\hline สิ & {$\left[\begin{array}{cc}100 & -29 \\
-29 & 9\end{array}\right]$} & {$\left[\begin{array}{c}-2.94 \\
0.9\end{array}\right]$} & {$\left[\begin{array}{c}\sin \theta+C_{\mu} \cos \theta \\
0.1\end{array}\right]$} \\
\hline & {$\left[\begin{array}{cc}96 & -49 \times 10^{3} \\
-49 \times 10^{3} & 1 \times 10^{6}\end{array}\right]$} & {$\left[\begin{array}{c}-4.9 \\
500\end{array}\right]$} & {$\left[\begin{array}{c}\sin \theta+C_{\mu} \cos \theta \\
0.5 \times 10^{-3}\end{array}\right]$} \\
\hline
\end{tabular}
propelled by a $1 \mathrm{kN}$ force

The introduction of supervisory control methods to the Parameter Error Algorithm is shown to effectively reduce the effect of data with errors, offsets or high levels of uncertainty associated with it.

\section{B. Influence of Additional Disturbances}

One area with potential for significant further work in the field of vehicle model identification is the development of methods to account for tyre-surface interaction and the potential colouring effect this has on the estimates of certain other parameters.

An example of this is illustrated by the 'humpback bridge' track depicted in Figure 13 consists of a short, steep, up and down, which is very exciting for the estimation algorithm. The mass estimate for the majority of this duty cycle is quite consistent, though some drift is observed. However, the initial approach to the humpback bridge loop was over a 'washboard' road with lots of very small severe undulations 


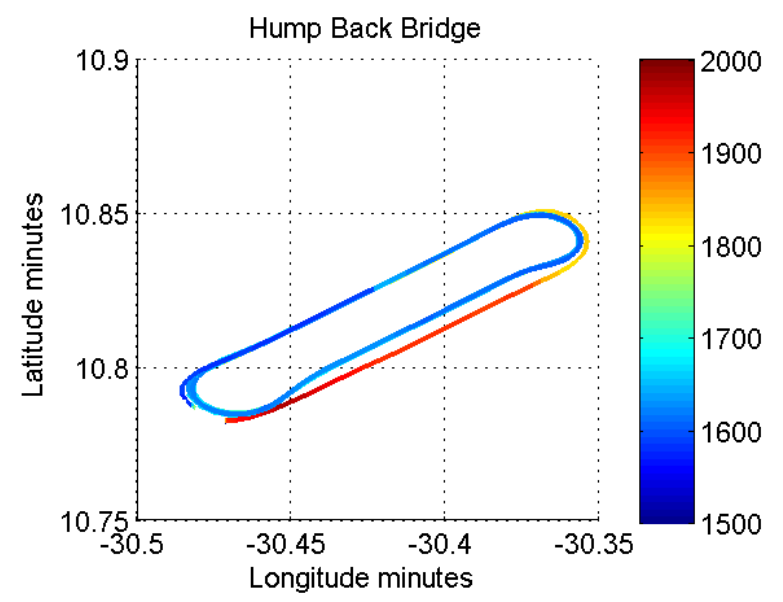

Fig. 13: Route map of the 'Hump back bridge' at JLR Gaydon. Multiple passes of the bridge section (top left straight) were made. Most return loops were via a flat road with axle articulation humps, but the first approach was made via an artificial 'washboard' surface (bottom right)

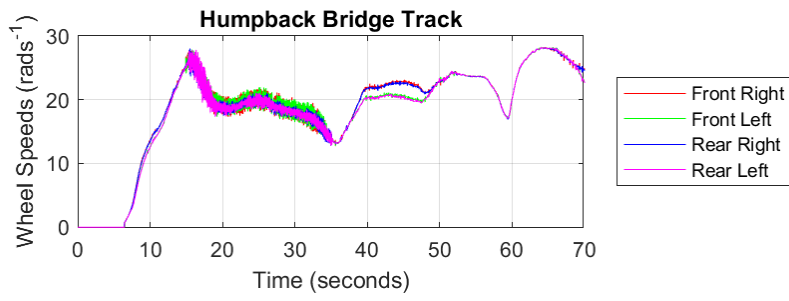

Fig. 14: All four wheelspeeds during the data collection activity of the 'Hump-Back Bridge' track at Gaydon showing the approach, first corner, and first pass of the bridge, notice the noise caused by the washboard surface which translates as additional rolling resistance and thus mass.

in close succession, adding a large amount of resistance to the vehicle through energy dissipated by tyres and suspensions. This 'washboard' is clearly visible in the speed trace at the beginning of Figure 14. Since the estimator has no model for this, it converged on a sensible mass on the otherwise flat road, which, once the extra energy loss was removed by returning to smooth tarmac, caused the mass estimate to drop to somewhat below its true value, and remain there consistently throughout the rest of the duty cycle.

Another example of this phenomenon is given in Figure 15. This is a track consisting of several different types of unmetalled road surface including hardpacked dirt and flints. The mass estimate was observed to be influenced by the road surfaces as they offer very different rolling friction coefficients. This may be clearly seen on the parallel out and return legs in the figure.

\section{Future Research}

Appropriate utilisation of the effect illustrated by the washboard road and varied un-metalled surfaces discussed in the

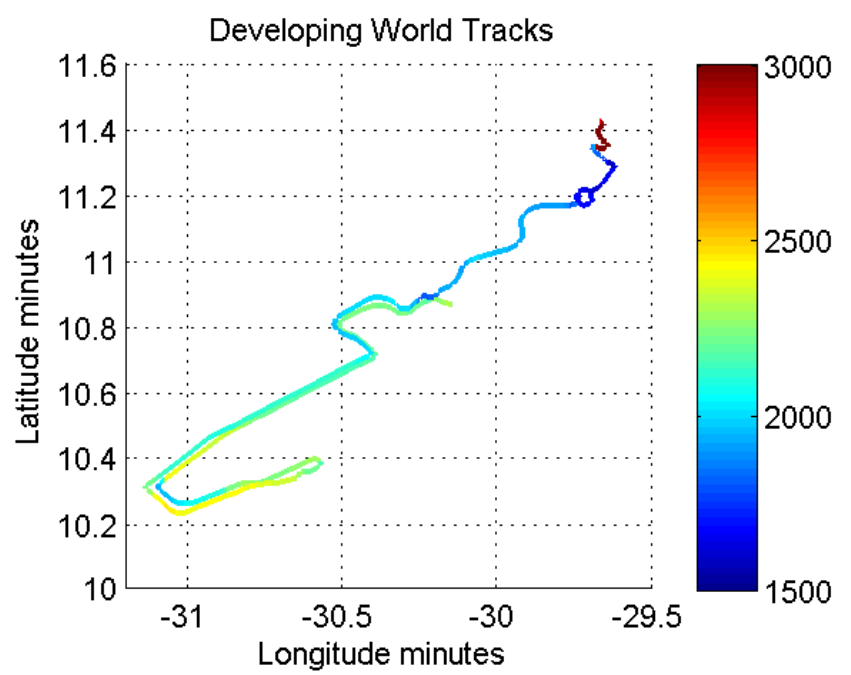

Fig. 15: Route map of the 'Developing world roads' track at JLR Gaydon.Several long passages of settled mass estimate at different values are evident where the road surfaces change

previous section may allow the estimation of a parameter describing road-tyre interaction. For example, if the mass is known not to change, this could be used to determine a coefficient to describe energy lost to the road surface, with obvious implications for vehicle energy management.

Additional work that suggests itself from the work conducted using the estimation techniques developed in this paper is the introduction of a like-parameterised data-fusion scheme for vehicle mass based on the vertical dynamics of the vehicle, in a similar vein to [20] or [6]. Another strand of work that requires addressing is the quantification of combined measurement uncertainty for the complete estimation scheme with the likeparameterised estimation scheme and supervisors in operation.

\section{Conclusions}

In this paper, the authors present several methods to practically improve the robustness of a parameter-error driven estimation algorithm applied to a vehicle parameter estimation case study. The paper provides the theoretical background to this algorithm and develops the need for both multiple data sources and selective estimation procedures when dealing with real world phenomena. Proposals of this type are explained and examples of their positive impact on the estimation performance are given.

The case study is concerned with the estimation of road gradient and vehicle mass, using only available data on the vehicle's networks. In this case the estimation algorithm takes powertrain torque, estimated braking torque and vehicle speed as inputs. The data fusion method introduced by the current authors in [25] is utilised for combining an accelerometer reading with the rest of the estimator. A supervisory-control inspired estimation management system is used to help eliminate innaccuracies introduced by certain systems such as friction brakes when they are activated.

The performance of the improved algorithm is compared with 
formulations of the state of the art in parameter estimation, namely the RLS and Kalman Filter methods. The ability of the improved parameter error driven estimation scheme for determining vehicle mass form available powertrain data is significantly better than for the state of the art, accurately identifying changes in vehicle mass from relatively poor quality input data. For gradient estimation or estimation of multiple parameters, the parameter-error driven scheme has already been demonstrated to be very effective.

Work remains to be done developing the mass estimation algorithm. At present its output is heavily influenced by certain environmental factors, including road geography. These influences are, however, clearly repeatable and using identical input data from the same duty cycles, the differentiation between vehicles ballasted to differing levels was consistent and accurate, in contrast to the current state of the art methods when provided with the same information.

\section{ACKNOWLEDGMENTS}

The authors would like to acknowledge the financial and in kind support of Jaguar Land Rover research in this project. Additionally the authors would like to thank FlandersDrive and the E-VECTOORC research consortium ${ }^{6}$ for facilitating some of the on-track testing of estimation algorithms.

\section{REFERENCES}

[1] Francois Caron, Emmanuel Duflos, Denis Pomorski and Philippe Vanheeghe GPS/IMU Data Fusion using Multisensor Kalman Filtering: Introduction of Contextual Aspects Journal of Information Fusion, Elsevier Science, July 2004

[2] Hosam K. Fathy, Dongsoo Kang and Jeffrey L Stein Online Vehicle Mass Estimation Using Recursive Least Squares and Supervisory Data Extraction Proceedings of the 2008 American Control Conference, Seattle WA. June 2008

[3] Jyotishman Ghosh, Stephane Foulard and Rafael Fietzek Vehicle Mass Estimation from CAN Data and Drivetrain Torque Observer SAE Technical Paper 2017-01-1590, 2017

[4] Henrik Jansson, Ermin Kozica, Per Sahlholm and Karl Henrik Johansson Improved road grade estimation using sensor fusion Reglermte Conference, Stockholm, Sweden, May 2006

[5] Kidambi, Narayanan and Harne, R. L. and Fujii, Yuji and Pietron, Gregory M. and Wang, K. W., emphMethods in Vehicle Mass and Road Grade Estimation SAE International Journal of Passenger Cars - Mechanical Systems, 2014

[6] Daeil Kim, Seibum B. Choi and Jiwon Oh Integrated Vehicle Mass Estimation Using Longitudinal and Roll Dynamics 12th International Conference on Control, Automation and Systems, Jeju Island, Korea, October 2012.

[7] I.D. Landau, R. Lozano, M. M'Saad Adaptive Control Springer-Verlag, London. 1998

${ }^{6}$ FP7/2007-2013 grant agreement No. 284708
[8] Boyuan Li, Jiawei Zhang, Haiping Du and Weihua Li Twolayer structure based on adaptive estimation for vehicle mass and road slope under longitudinal motion

[9] Peter Lingman and Bengt Schmidtbauer Road Slope and Vehicle Mass Estimation Using Kalman Filtering Vehicle System Dynamics, 37:sup1, 12-23, Taylor and Francis 2002.

[10] Mahyuddin, Muhammad N; Na, J; Herrmann, G; Ren, $\mathrm{X}$ and Barber, P. An Adaptive Observer-based parameter estimation algorithm with application to Road Gradient and Vehicles Mass Estimation IEEE UKACC International Conference on Control 2012. IEEE Control Systems Society, 2012

[11] Mahyuddin, Muhammad N; Na, J; Herrmann, G; Ren, X and Barber, P. Adaptive Observer-based parameter estimation with application to Road Gradient and Vehicle Mass Estimation. IEEE Transactions on Industrial Electronics, 2013.

[12] Michael L. McIntyre, Tejas J. Ghotikar, Ardalan Vahidi, Xubin Song and Darren M. Dawson A Two-Stage Lyapunov-Based Estimator for Estimation of Vehicle Mass and Road Grade IEEE Transactions on Vehicular Technology Vol. 58, No. 7, September 2009

[13] W. Milliken and D. Milliken Race Car Vehicle Dynamics SAE Inc. Detroit MI, 1995.

[14] Na, J; Herrmann, G; Ren, Xuemei; Mahyuddin, Muhammad N and Barber, P. Robust Adaptive Finite-Time Parameter Estimation and Control of Nonlinear Systems. 2011 IEEE Multi-Conference on Systems and Control, Denver. 2011.

[15] Na, J; Mahyuddin, Muhammad N; Herrmann, G; Ren, Xuemei and Barber, P. Robust adaptive finite-time parameter estimation and control for robotic systems International Journal of Robust and Nonlinear Control 2015, Volume 25, Issue 16, pages 3045-3071.

[16] Ying Kun Peng and M.F. Golnaraghi A Vector-Based Gyro-Free Inertial Navigation System by Integrating Existing Accelerometer Network in a Passenger Vehicle Position Location and Navigation Symposium, IEEE, 2004

[17] Enrico Raffone Road Slope and Vehicle Mass Estimation for Light Commercial Vehicle using Linear Kalman Filter and RLS with forgetting factor integrated approach 16th International Conference on Information Fusion, Istanbul, Turkey, IEEE, 2013

[18] R. Rajamani Vehicle Dynamics and Control Springer, New York, 2006

[19] Rajesh Rajamani, Damrongrit Piyabongkarn, Vasilis Tsourapas and Jae Y. Lew Parameter and State Estimation in Vehicle Roll Dynamics IEEE Transaction on Intelligent Transportation Systems, Vol. 12, No. 4, December 2011

[20] Maryam Sadeghi Reineh, Martin Enqvist and Fredrik Gustafsson Detection of Roof Load for Automotive Safety Systems 52nd IEEE Conference on Decision and Control, Florence, Italy, December 2013.

[21] Per Sahlholm and Karl Henrik Johansson Road grade estimation for look-ahead vehicle control using multiple measurement runs IEEE Journal of Control Engineering Practice, vol. 18, pp. 1328-1341, IEEE, 2010 
[22] Per Sahlholm and Karl Henrik Johansson Segmented road grade estimation for fuel efficient heavy duty vehicles proceedings of 49th IEEE Conference on Decision and Control, 2010

[23] Dan Simon Optimal State Estimation: Kalman, $H_{\infty}$, and Nonlinear Approaches John Wiley and Sons, 2006

[24] Salah Sukkarieh, Eduardo M. Nebot and Hugh F. Durrant-Whyte Achieving Integrity in an INS/GPS Navigaion Loop for Autonomous Land Vehicle Applications Proceedings of the 1998 IEEE International Conference on Robotics \& Automation, Leuven, Belgium, may 1998

[25] Wragge-Morley, R; Herrmann, G; Barber, P and Burgess,

$\mathrm{S}$. Information fusion for vehicular systems parameter estimation using an extended regressor in a finite time estimation algorithm IEEE UKACC International Conference on Control 2014. IEEE Control Systems Society, 2014

[26] Wragge-Morley, R; Herrmann, G; Barber, P and Burgess, S. Gradient and Mass Estimation from CAN based data for a light passenger car SAE International Journal of Passenger Cars Electronic and Electrical Systems, May 2015

[27] Ardalan Vahidi, Maria Druzhininia, Anna Stefanopolou, Huei Peng Simultaneous Mass and Time-Varying Grade Estimation for Heavy-Duty Vehicles Proceedings of the American Control Conference, Denver Colorado, June 2003

The methods used for comparison between the novel method and the state of the art are presented here for the sake of completeness

\section{APPENDIX A}

PROOF OF STABILITY FOR REGRESSOR-BASED DATA FUSION METHOD

The proof of stability is split into two parts, Part A deals with the proof of stability of the adaptive observer, while Part B discusses the stability of the supervised Kalman filter structure.

Part $\mathbf{A}$ of the proof of stability largely adopts the method established in [11] with some simplifications for brevity and modifications to account for the aspect of data fusion in (18) and (19). For simplicity, only the velocity dynamics (6) and the relevant observer are investigated. Here $A_{21}=A_{22}=0$ so that the system dynamics satisfy $\dot{x}_{2}=B_{12} u_{11}+\bar{B}_{2} \phi \Theta+\zeta_{2}$. Hence, the observer dynamics are $\dot{\hat{x}}_{2}=B_{12} u_{11}+\bar{B}_{2} \phi \hat{\Theta}-$ $L_{2}\left(y-\hat{x}_{2}\right)$ and the error dynamics $\dot{\tilde{x}}_{2}=\bar{B}_{2} \phi \tilde{\Theta}-L_{2}\left(\tilde{x}_{2}\right)$, where $\tilde{x}_{2}=\hat{x}_{2}-x_{2}$. Considering these dynamics, we now employ a Lyapunov function:

$$
V=\frac{1}{2} \tilde{x}_{2} P \tilde{x}_{2}+\frac{1}{2} \tilde{N}_{N}^{T} M_{N}^{-1} \Gamma^{-1} M_{N}^{-1} \tilde{N}_{N}
$$

where the following structural constraint is raised $P \bar{B}_{2}=F$. An important assumption for the Lyapunov function (25) to exist is $\min \left\{\operatorname{eig}\left(M_{N}\right)\right\}>0$ so that $M_{N}$ remains invertible. In [14][15], it has been shown that $M_{N}$ remains invertible or $\min \left\{\operatorname{eig}\left(M_{N}\right)\right\}>0$ if (and only if) $\phi_{f N}$ is persistently excited. Thus, to achieve a proof of stability for algorithm convergence with this Lyapunov function, persistent excitation of $\phi_{f N}$ is necessary.
Differentiating the Lyapunov function with respect to time provides:

$$
\begin{aligned}
\dot{V}= & \tilde{x}_{2} P \dot{\tilde{x}}_{2}+\frac{1}{2} \frac{d}{d t}\left(\tilde{N}_{N}^{T} M_{N}^{-1} \Gamma^{-1} M_{N}^{-1} \tilde{N}_{N}\right) \\
= & \tilde{x}_{2} P \dot{\tilde{x}}_{2}+\tilde{N}_{N}^{T} M_{N}^{-1} \Gamma^{-1} \frac{d}{d t}\left(M_{N}^{-1} \tilde{N}_{N}\right) \\
= & \tilde{x}_{2} P\left(\bar{B}_{2} \phi \tilde{\Theta}-L_{2}\left(\tilde{x}_{2}\right)\right) \\
& +\tilde{N}_{N}^{T} M_{N}^{-1} \Gamma^{-1} \frac{d}{d t}\left(M_{N}^{-1} \tilde{N}_{N}\right)
\end{aligned}
$$

Note that

$$
M_{N}^{-1} \tilde{N}_{N}=\tilde{\Theta}-M_{N}^{-1} \xi_{\phi N f}
$$

and $\frac{d}{d t}\left(M_{N}^{-1} \tilde{N}_{N}\right)=\dot{\tilde{\Theta}}-M_{N}^{-1} \dot{M}_{N} M_{N}^{-1} \xi_{\phi N f}-M_{N}^{-1} \dot{\xi}_{\phi N f}$. Here, $\xi_{\phi c}=-M_{N}^{-1} \dot{M}_{N} M_{N}^{-1} \xi_{\phi N f}-M_{N}^{-1} \dot{\xi}_{\phi N f}$ which is bounded since all terms in (20) and (22) remain bounded. Hence, this provides the following result:

$$
\begin{aligned}
\dot{V}= & \tilde{x}_{2} P\left(\bar{B}_{2} \phi \tilde{\Theta}-L_{2} \tilde{x}_{2}\right)+\tilde{N}_{N}^{T} M_{N}^{-1} \Gamma^{-1}\left(\dot{\tilde{\Theta}}+\xi_{\phi c}\right) \\
= & \tilde{x}_{2} P\left(\bar{B}_{2} \phi \tilde{\Theta}-L_{2} \tilde{x}_{2}\right)+\tilde{N}_{N}^{T} M_{N}^{-1} \phi^{T} F\left(y-\hat{x}_{2}\right) \\
& -\tilde{N}_{N}^{T} \omega_{1} \Omega \frac{\tilde{N}}{\tilde{N}}-\tilde{N}_{N}^{T} \omega_{2} \Omega \tilde{N}+\tilde{N}_{N}^{T} M_{N}^{-1} \Gamma^{-1} \xi_{\phi c}
\end{aligned}
$$

Hence, considering again (27), it follows:

$$
\begin{aligned}
\dot{V}= & -\tilde{x}_{2} P L_{2} \tilde{x}_{2}-\xi_{\phi N f} M_{N}^{-1} \phi^{T} F \tilde{x}_{2}-\tilde{N}_{N}^{T} \omega_{1} \Omega \frac{\tilde{N}}{\tilde{N}} \\
& -\tilde{N}_{N}^{T} \omega_{2} \Omega \tilde{N}+\tilde{N}_{N}^{T} M_{N}^{-1} \Gamma^{-1} \xi_{\phi c}
\end{aligned}
$$

Choosing $L_{2}$ positive it follows employing the smallest eigenvalue $\min \{\operatorname{eig}(\Omega)\}$ :

$$
\begin{aligned}
\dot{V} \leq & -\left\|\tilde{x}_{2}\right\|^{2} P L_{2}-\xi_{\phi N f} M_{N}^{-1} \phi^{T} F \tilde{x}_{2} \\
& -\left\|\tilde{N}_{N}^{T}\right\| \omega_{1} \min \{\operatorname{eig}(\Omega)\} \\
& -\left\|\tilde{N}_{N}\right\|^{2} \omega_{2} \min \{\operatorname{eig}(\Omega)\}+\tilde{N}_{N}^{T} M_{N}^{-1} \Gamma^{-1} \xi_{\phi c}
\end{aligned}
$$

Now the inequalities

$$
\xi_{\phi N f} M_{N}^{-1} \phi^{T} F \tilde{x}_{2} \leq \frac{c_{x}}{2} \tilde{x}_{2}^{2}+\frac{1}{2 c_{x}}\left\|\xi_{\phi N f} M_{N}^{-1} \phi^{T} F\right\|^{2}
$$

and

$$
\tilde{N}_{N}^{T} M_{N}^{-1} \Gamma^{-1} \xi_{\phi c} \leq \frac{c_{N}}{2}\left\|\tilde{N}_{N}\right\|^{2}+\frac{1}{2 c_{N}}\left\|M_{N}^{-1} \Gamma^{-1} \xi_{\phi c}\right\|^{2}
$$

for sufficiently small values of $c_{x}>0$ and $c_{N}>0$ are used:

$$
\begin{aligned}
\dot{V} \leq & -\left\|\tilde{x}_{2}\right\|^{2}\left(P L_{2}-\frac{c_{x}}{2}\right)-\left\|\tilde{N}_{N}^{T}\right\| \omega_{1} \min \{\operatorname{eig}(\Omega)\} \\
& -\left\|\tilde{N}_{N}\right\|^{2}\left(\omega_{2} \min \{\operatorname{eig}(\Omega)\}-\frac{c_{N}}{2}\right) \\
& +\frac{1}{2 c_{x}}\left\|\xi_{\phi N f} M_{N}^{-1} \phi^{T} F\right\|^{2}+\frac{1}{2 c_{N}}\left\|M_{N}^{-1} \Gamma^{-1} \xi_{\phi c}\right\|^{2}
\end{aligned}
$$

so that $\left(P L_{2}-\frac{c_{x}}{2}\right)>0$ and $\left(\omega_{2} \min \{\operatorname{eig}(\Omega)\}-\frac{c_{N}}{2}\right)>0$. The final inequality (29) requires the last two terms to remain bounded. Once this holds the parameter estimation scheme can be proven to be ultimately bounded stable, i.e. the estimator guarantees convergence to a bounded set close to the true 
parameter values. (See further detail in [11][14][15]).

The boundedness of the last two terms in (29) can be satisfied: For matrix $M_{N}$ the following has to hold $\min \left\{\operatorname{eig}\left(M_{N}\right)\right\}>c_{M}>0$ for some small value $c_{M}>0$, which can be guaranteed by a certain level of persistent excitation [11][14][15]. Moreover, the term $\phi_{f N}$ remains bounded if we consider that all elements in $\phi_{f N}$ remain practically bounded. For instance, from (19) and (4a), the value of the net-force $F_{n e t}$ remains bounded, while $g$ is constant at all times.

Part B considers that the resulting estimate $\hat{\Theta}$ is further processed through the suggested Kalman filtering scheme as discussed in Subsection III-A. (Note that the employed Kalman filter is designed under the assumption of a constant signal output.) The Kalman filter without supervisor is exponentially stable. When subjected to the mechanism of halting the filter state and subsequently continuing the filtering process after re-instantiation, this stability is retained despite the periods where the Kalman filter is halted. Since the Kalman filter and the parameter error estimation scheme are in series, the overall stability is determined by the stability of the single elements.

\section{APPENDIX B \\ Continuous-time Recursive Least Squares}

Least squares estimation relies on minimising the square of the errors between some measured data and some calculated estimate. For some system whose model has been parameterised to:

$$
\begin{aligned}
& \boldsymbol{x}(t)=\boldsymbol{\varphi} \boldsymbol{\Theta}+B \boldsymbol{u}+\zeta \\
& \boldsymbol{y}(t)=C \boldsymbol{x}(t)+\boldsymbol{v}
\end{aligned}
$$

where $\boldsymbol{x}$ are the states of the system, $\boldsymbol{\varphi}$ is the signal matrix and $\Theta$ is a vector of plant parameters which are not known. The 'prediction' of $\boldsymbol{y}$ is based on this linear plant model and an estimated set of parameters, $\hat{\Theta}$.

$$
\begin{aligned}
& \hat{\boldsymbol{x}}(t)=\boldsymbol{\varphi}(t) \hat{\boldsymbol{\Theta}}+B \boldsymbol{u}+\zeta \\
& \hat{\boldsymbol{y}}(t)=C \hat{\boldsymbol{x}}(t)+\boldsymbol{v}
\end{aligned}
$$

We wish to minimise a 'cost' function of the form:

$$
J=\int_{0}^{t} \boldsymbol{\epsilon}_{y}^{T} \epsilon_{y} d r
$$

where:

$$
\epsilon_{y}=\boldsymbol{y}-\varphi \hat{\Theta}
$$

which gives a parameter update law of the form:

$$
\dot{\hat{\boldsymbol{\Theta}}}=P(t) \boldsymbol{\varphi}^{T}(t) \boldsymbol{\epsilon}_{y}(t)
$$

with the gain update law:

$$
\dot{P}(t)=\lambda(t) P-P \varphi^{T}(t) \varphi(t) P
$$

The RLS mass estimator is based on the standard parameterisation shown in equation (2) but with the terms effectively swapped so that the unknown term is now $\frac{1}{m}$ (shown in equation (36)). The input term $B_{1} \mathbf{u}_{1}$ is now used to include the gradient as a 'known' input $-g\left(\sin \theta_{R}+C_{\mu} \cos \theta_{R}\right)$.

$$
\phi \Theta=F_{n e t}\left(\frac{1}{m}\right)
$$

Practically this situation could be achieved by running parallel single parameter estimators. This type of method is often employed in the literature [21] [17] [8].

\section{APPENDIX C}

\section{Kalman Filter Mass Estimation Design}

Another popular estimation method in the literature is the Kalman Filter. A Kalman filter to estimate vehicle mass only can be formulated using the system of equations proposed in 41 .

In order to use Kalman filtering to estimate vehicle parameters like the other estimation methods discussed in this chapter, the dynamics and subsequent estimation problem must be reformulated so that the parameter to be estimated becomes a state.

The Kalman filter formulation employed is in continuous time, as presented by Simon [23]. The estimated parameter is treated as a state in the filter formulation and this state is assumed to have dynamics of the form:

$$
\begin{array}{r}
\dot{\mathbf{x}}_{m}=A \mathbf{x}+B_{m} \mathbf{u}+\mathbf{w} \\
\mathbf{y}=C \mathbf{x}+\mathbf{v}
\end{array}
$$

where $\mathbf{w}$ and $\mathbf{v}$ are continuous time white noise processes with covariances $Q_{c}$ and $R_{c}$ :

$$
\begin{aligned}
& \mathbf{w} \sim\left(0, Q_{c}\right) \\
& \mathbf{v} \sim\left(0, R_{c}\right)
\end{aligned}
$$

The state $\mathbf{x}_{m}=\left[\begin{array}{c}v_{\text {measured }} \\ \frac{1}{m}\end{array}\right]$ and the gradient terms are treated as an input $\boldsymbol{u}_{m}=\left[\begin{array}{c}\sin \theta+C_{\mu} \cos \theta \\ 0\end{array}\right]$ such that the overall process for the vehicle mass estimation problem may be written:

$$
\begin{aligned}
& \dot{\mathbf{x}}=\underbrace{\left[\begin{array}{cc}
0 & F_{n e t} \\
0 & 0
\end{array}\right]}_{A} \mathbf{x}+B \underbrace{\left[\begin{array}{c}
-g\left(\sin \theta+C_{\mu} \cos \theta\right) \\
0
\end{array}\right]}_{\mathbf{u}}+\underbrace{\left[\begin{array}{c}
\zeta_{1} \\
\zeta_{2}
\end{array}\right]}_{\mathbf{w}} \\
& \mathbf{y}=\underbrace{\left[\begin{array}{ll}
1 & 0 \\
0 & 1
\end{array}\right]}_{C} \mathbf{x}+\mathbf{v}
\end{aligned}
$$

where the process noises $\zeta$ are assumed to be adequately represented by $\boldsymbol{w}$. The state and covariance of the continuous time Kalman filtering process are initialised by

$$
\begin{aligned}
\hat{\boldsymbol{x}}_{m}(0) & =E\left[\boldsymbol{x}_{m}(0)\right] \\
P(0) & =E\left[\left(\boldsymbol{x}_{m}(0)-\hat{\boldsymbol{x}}_{m}(0)\right)\left(\boldsymbol{x}_{m}(0)-\hat{\boldsymbol{x}}_{m}(0)\right)^{T}\right]
\end{aligned}
$$

where the notation $E\left(x_{i}\right)$ denotes the expected value of variable $x_{i}$. The covariance is propagated according to the rule in equation (44): 


$$
\dot{P}=-P C_{m}^{T} R_{c}^{-1} C_{m} P+A_{m} P+P A_{m}^{T}+Q_{c}
$$

and the estimated state is propagated by equation (45):

$$
\dot{\hat{\boldsymbol{x}}}_{m}=A_{m} \hat{\boldsymbol{x}}_{m}+K\left(\boldsymbol{y}_{m}-C_{m} \hat{\boldsymbol{x}}_{m}\right)
$$

where the Kalman gain $K$ is

$$
K=P C_{m}^{T} R_{c}^{-1}
$$

TABLE III: Noise Covariance models used for individual parameter Kalman Filtering. The noise model is given as a vector of covariances for the measured state in each case; the state vectors are defined in equation (37) and (38) for mass only estimation. Systematic noise $\mathbf{w}$ represents unmodelled physical deviation in the plant and is part of the state equation, whilst measurement noise $\mathbf{v}$ describes some model of sensor noise. These vectors are used to define the noise covariance matrices $R$ and $Q$ respectively.

\begin{tabular}{|c|c|}
\hline Systematic Noise & {$\left[\begin{array}{c}0.01 \\
1\end{array}\right]$} \\
\hline Measurement Noise & {$\left[\begin{array}{c}0 \\
0.01\end{array}\right]$} \\
\hline
\end{tabular}

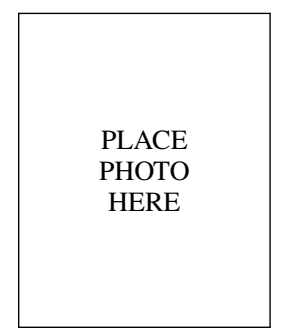

Robert Wragge-Morley received his degree in Mechanical Engineering and $\mathrm{Ph} . \mathrm{D}$ in the same subject from the University of Bristol, U.K. in 2012 and 2017 respectively. He has subsequently worked as a Research Associate within that University's Electrical Energy Management Group before moving to the Powertrain and Vehicle Research Centre at the University of Bath in 2018. His current research interests are in vehicle self system awareness and prediction, human vehicle interaction and real driving emissions (RDE).

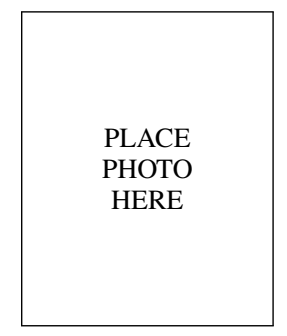

Guido Herrmann received his $\mathrm{Ph}$. D. degree from University of Leicester, UK, in 2001. From 2001 to 2003, he was a Senior Research Fellow in the Data Storage Institute in Singapore. From 2003 until 2007, he was a research associate, fellow, and lecturer in the University of Leicester. He joined the University of Bristol, UK, as a lecturer in March 2007. He was promoted to a Senior Lecturer in 2009 and a Reader in Control and Dynamics in 2012. $\mathrm{He}$ is a Senior Member of the IEEE. His research interest covers the development and application of novel, robust and nonlinear control systems. Dr. Herrmann was appointed a Professor of Robotics and Intelligent Control at the University of Manchester in 2019 .

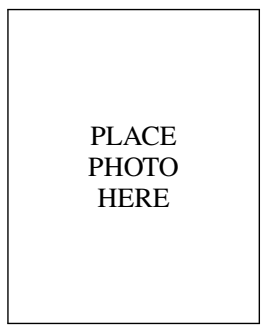

Phil Barber received his degree in Electrical and Control Engineering at Birmingham University. After a year at GECs Hirst Research Centre, he joined the Automotive industry in 1985 at the Lucas Research Centre. He gained his Phd in 1992 from the University of Warwick researching fuel injected engines. Joining Jaguar in 1990, he went on to be part of the team working on steer by wire, lane keeping and adaptive cruise control (ACC) demonstrators for the PROMETHEUS project. The ACC won the Henry Ford Technical Award, the highes award for technical achievement in the Ford Motor Corporation in 2000. Then, he oversaw the changeover of military standard ACC radar manufacture to automotive production standards and he lead the ACC delivery team. He returned to Research in 2008 as the Technical Specialist for Chassis Systems and Vehicle Capability. Current interests include vehicle dynamics, state estimation, distributed and networked systems for vehicle control and regenerative braking. 